\title{
Efecto de la tintura de Propóleo vs Clorhexidina en el tratamiento de la Enfermedad Periodontal en caninos domésticos
}

\author{
Effect of Propolis dye vs Chlorhexidine in the treatment of Periodontal Disease in \\ domestic canines
}

\author{
Diego Medina-Valarezo ${ }^{1,2 *}$ iD, Martha Camacho ${ }^{\text {(D) }}$, Manuel García-Herreros ${ }^{3}$ (D), Andrés Ortega-Ojeda1 iD y \\ Francisco Angulo-Cubillán ${ }^{4,5}$
}

${ }^{1}$ Grupo de Investigación de Estudios de Bienestar Animal, Facultad Ciencias de la Salud Eugenio Espejo. Universidad UTE, Sede Occidental, Quito, Ecuador. ${ }^{2}$ Hospital Veterinario Planeta Vida. Latacunga, Ecuador. ${ }^{3}$ Instituto Nacional de Investigação Agrária e Veterinária, I. P. (INIAV, I.P.). Santarém, Portugal. ${ }^{4}$ Grupo de Investigación de Salud, Producción y Biotecnología Animal, Facultad Ciencias de la Salud Eugenio Espejo. Universidad UTE. Sede Santo Domingo, Ecuador. ${ }^{5}$ Departamento de Sanidad Animal, Facultad de Ciencias Veterinarias, Universidad del Zulia. Maracaibo, Venezuela.

*Correo electrónico: diego.medina@ute.edu.ec

\section{RESUMEN}

En el presente estudio se analizó el efecto de la tintura de propóleo (TP) vs clorhexidina (C) en el tratamiento de la enfermedad periodontal en la especie canina (EPC). Para ello se llevó a cabo la aplicación terapéutica en 30 pacientes con diferentes grados de enfermedad periodontal (leve, media y grave). Se tomaron muestras de la cavidad bucal para el cultivo posterior e identificación de los microorganismos patógenos implicados en el proceso patológico. Se identificaron 4 géneros de patógenos: Staphylococcus spp., Corynebacterium spp., Lactobacillus spp. y Enterobacterias. El porcentaje de casos que respondieron al tratamiento fue 80 y $3,4 \%$ para TP y $C$, respectivamente. $C$ no demostró tener ninguna acción eficiente en el tratamiento de EPC. Sin embargo, TP mostró ser el tratamiento de elección, independientemente del patógeno, asociación de éstos o el grado de progresión de la EPC.

Palabras clave: Efecto bactericida; propóleo; clorhexidina; enfermedad periodontal; canino

\begin{abstract}
In the present study the effect of propolis tincture (PT) vs chlorhexidine $(C)$ as a treatment of canine periodontal disease (CPD) was analysed. A therapeutic application in 30 patients with different degrees of CPD was carried out (low, moderate and severe). Samples from the oral cavity were collected for culture and further identification of pathogenic microorganisms involved in the pathological process. Four pathogens of different genera were identified; Staphylococcus spp., Corynebacterium spp., Lactobacillus spp. and Enterobacterias. The cases that responded to treatment were 80 and $3.4 \%$ for PT and C, respectively. C did not demonstrate any efficient action in the treatment of CPD. However, PT demonstrated to be the treatment of choice regardless the pathogen, pathogen associations or the degree of progression of CPD.
\end{abstract}

Key words: Bactericidal effect; propolis; chlorhexidine; periodontal disease; canine 


\section{INTRODUCCIÓN}

La Enfermedad Periodontal Canina (EPC) es la patología oral más común que afecta a los caninos domésticos (Canis lupus familiaris), asociada a problemas sistémicos [15]. Los factores que predisponen la EPC son: edad, especie, raza, genética, conducta de masticación, dieta, oclusión, estado general de salud, cuidados caseros, frecuencia de atención odontológica y la flora bacteriana, entre otros [5,7]. Aunque las bacterias no siempre son los agentes etiológicos primarios, sin embargo, son las causantes de perpetuar la EPC causada por malos hábitos alimenticios o descuidos en el cuidado de la salud oral de las mascotas [4].

Se ha reportado que la EPC se clasifica en etapas progresivas, que pueden describirse en grados de acuerdo con la gravedad y a la extensión de las zonas afectadas [18], cuya evolución comprende estadios: Grado 1 (Leve) Gingivitis, acúmulo de sarro evidente y retracción de la encía en $25 \%$ : Grado 2 (Moderado) placa de sarro que cubre casi todo el diente y retracción de la encía en 50 \%; Grado 3 (Grave) exposición de raíces dentales, movimiento y pérdida de piezas dentales [3].

Tradicionalmente se ha tratado la EPC con soluciones antisépticas, antibióticos y medicina natural, no obstante, el uso indiscriminado de éstas y la baja eficacia de los protocolos terapéuticos, manifestados en recidivas de la enfermedad, sugieren el uso de nuevos medicamentos que contribuyan al tratamiento de esta patología [14]. La medicina alternativa propone esquemas terapéuticos que reemplazan y contribuyen de forma concomitante al tratamiento de enfermedades causadas por bacterias. Productos naturales derivados de plantas medicinales han demostrado ser una fuente importante de compuestos biológicamente activos, como es el caso de Mangifera indica [12].

Sustancias derivadas de los animales, tales como la miel de abeja o la apitoxina, han sido reportados para el tratamiento de enfermedades causadas por bacterias [2, 8, 13, 19]. La Tintura de Propóleo (TP) es una sustancia resinosa producida por las abejas (Apis mellifera), para tapizar el interior de la colmena y protegerla de agentes infecciosos, por poseer efectos farmacológicos antimicrobianos, atribuidos a un compuesto activo conocidos como flavonoides [17]. Su composición y propiedades varían debido a la influencia geográfica donde se ubican las colmenas, así como de la riqueza en la flora predominante $[1,11]$

Existen estudios que respaldan el uso de TP "in vitro" sobre diferentes tipos de bacterias como Salmonella, Shiguella, Yersinia y Escherichia [6]. Esta sustancia ha sido utilizada en la aplicación terapéutica en diferentes patologías, tales como caries, aftas bucales y cicatrizaciones en el hombre [17]. El uso de TP en medicina veterinaria, no se encuentra ampliamente difundido.

El trabajo de investigación plantea la hipótesis de que TP actúa como antibiótico natural, reduciendo la flora bacteriana que causa EPC, por lo que se planteó el objetivo de determinar el efecto de TP en el tratamiento de la enfermedad, comparado con el tratamiento tradicional con Clorhexidina $(C)$ en la especie canina.

\section{MATERIALES Y MÉTODOS}

\section{Ubicación del estudio}

El estudio se llevó a cabo en pacientes del Hospital Veterinario Planeta Vida, en la ciudad de Latacunga, provincia Cotopaxi, Ecuador, la cual se encuentra en el centro-norte de la Región interandina, a una altitud de 2.750 metros sobre el nivel del mar (m.s.n.m.) [9].

\section{Unidades de estudio y técnica experimental}

Se realizó el análisis de los casos que acudían a consulta con cierto grado de EPC y se clasificaron 30 pacientes en tres grupos de la siguiente manera: Grado 1 (Leve) Gingivitis, acúmulo de sarro evidente y retracción de la encía en 25 \%: Grado 2 (Moderado) placa de sarro que cubre casi todo el diente, retracción de encía en 50 \%; Grado 3 (Grave) exposición de raíces dentales, movimiento y pérdida de piezas dentales [3] (FIG 1 y 2). Cada individuo fue tratado en el lado derecho con TP e izquierdo con $\mathrm{C}$.

\section{Toma de muestras}

Se realizaron las primeras tomas de muestra mediante hisopado del tejido contaminado para ser llevado inmediatamente al laboratorio, donde se le procesó para la identificación y cultivo respectivo. Posterior al tratamiento empleado (TP y C), se tomó y procesó una segunda muestra de la misma forma que la anterior. Las muestras fueron procesadas y los diagnósticos realizados por técnicas microbiológicas.

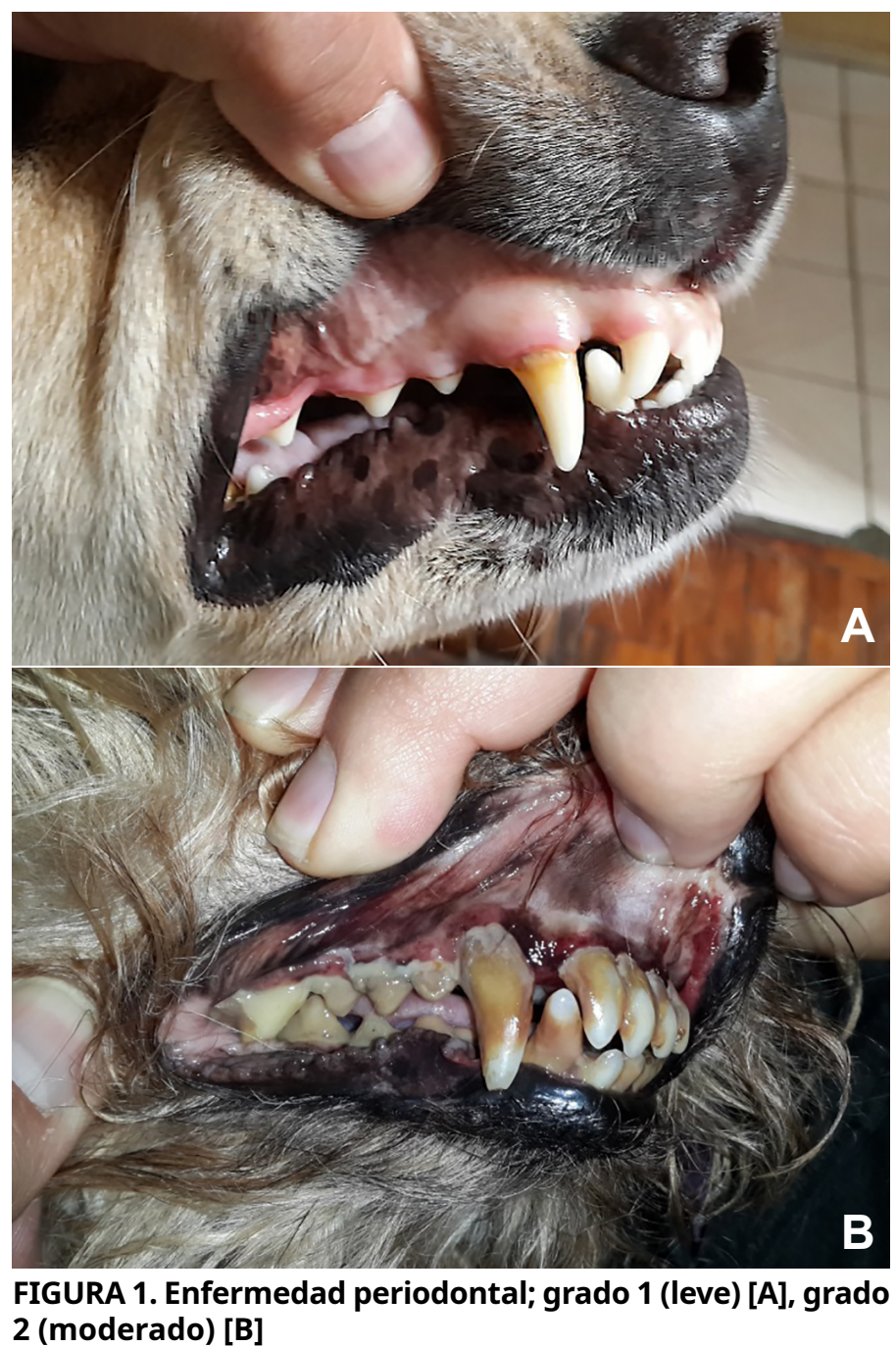




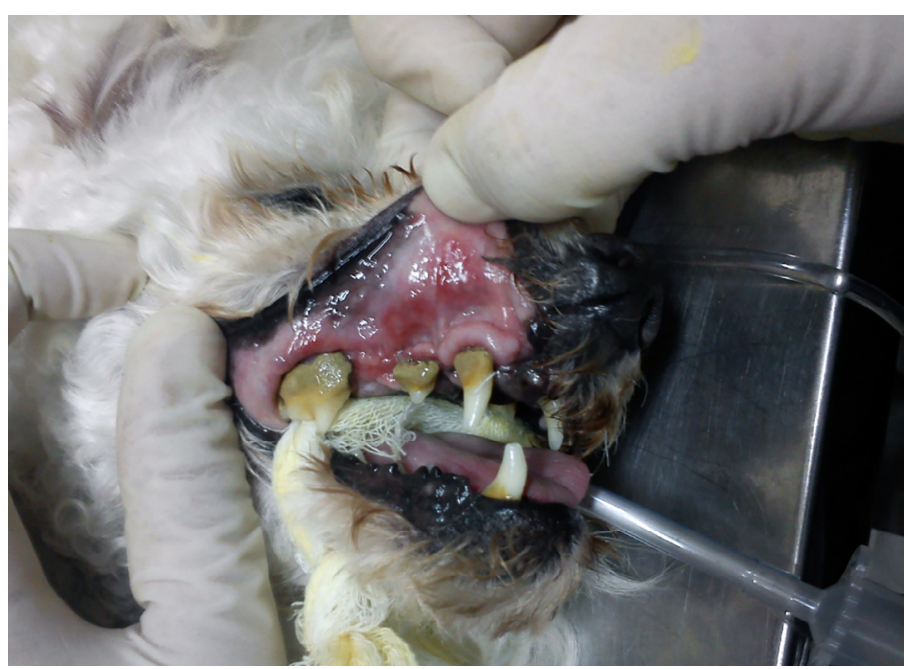

FIGURA 2. Enfermedad periodontal grado 3 (grave)

\section{Protocolo terapéutico}

Se incluyó el uso del equipo de ultrasonido odontológico (Jet Sonic B.P, Gnatus LTDA, Brasil), para el retiro del cálculo dental contaminado y se procedió a colocar el tratamiento con TP $20 \%$ o C $2 \%$, la solución antiséptica (TP al $20 \%$ y solución acuosa de $\mathrm{C}$ al $2 \%$ ) en las zonas afectadas, permitiendo que la solución ejerza su efecto realizando embrocaciones repetidas de ambos principios activos con gasa, utilizando el mismo paciente para ambos tratamientos.

\section{Análisis de datos}

El análisis se realizó con la base de datos del programa informático SPSS para Windows [20]. Para el análisis se utilizó la prueba Kolmogorov-Smirnov para conocer la normalidad de las variables, las medidas de dispersión normal: media, desviación estándar, intervalo de confianza al $95 \%$ para variables cuantitativas y porcentajes para variables cualitativas. Para comparar medias de variables paramétricas se utilizó la prueba t Student. Las frecuencias de distribución se compararon para grupos independientes. Valores de $P$ menores a 0,05 , fueron considerados estadísticamente significativos [20].

\section{RESULTADOS Y DISCUSIÓN}

Al describir los resultados correspondientes al crecimiento bacteriano en cultivo de cavidad oral en la especie canina, se observó una predominancia de Staphylococcus spp. sobre los demás gérmenes aislados $(P<0,05)$ (TABLA I). Además, se detectó como segundo microorganismo predominante a Corynebacterium spp. $(P<0,05)$. El tercero es la combinación de ambos gérmenes Staphylococcus spp. y Corynebacterium spp. encontrados en un mismo individuo $(P<0,05)$. El resto de los microorganismos aparecieron de forma esporádica, y por tanto su relevancia fue relativa.

En la TABLA II se observa diferencia estadística significativa, respecto al efecto positivo del TP sobre las bacterias aisladas en cultivos de pacientes caninos con EPC $(P<0,05)$. Sin embargo, TC en humanos proporcionan datos diferentes en cuanto a la EP,
TABLA I

Microorganismos y sus asociaciones, identificados en los pacientes sujetos a estudio $(n=30)$

\begin{tabular}{cc}
\hline Microorganismo & $\begin{array}{c}\text { Pacientes } \\
\text { diagnostico } \\
\text { positivo (\%) }\end{array}$ \\
\hline Staphylococcus spp. & $86,7^{\mathrm{a}}$ \\
Corynebacterium spp. & $36,7^{\mathrm{b}}$ \\
Lactobacillus spp. & $3,4^{\mathrm{c}}$ \\
Enterobacterias & $6,7^{\mathrm{c}}$ \\
$\begin{array}{c}\text { Staphylococcus spp. | Corynebacterium spp. } \\
\text { Staphylococcus spp. | Lactobacillus spp. }\end{array}$ & $16,6^{\mathrm{d}}$ \\
$\begin{array}{c}\text { Staphylococcus spp. | Enterobacterias } \\
\text { Staphylococcus spp. | Corynebacterium spp. }\end{array}$ & $3,33^{\mathrm{c}}$ \\
| Lactobacillus spp. & $0^{\mathrm{c}}$ \\
Staphylococcus spp. | Corynebacterium spp. & $3,33^{\mathrm{c}}$ \\
\hline Enterobacterias
\end{tabular}

Letras distintas indican diferencias estadísticamente significativas $(P<0,05)$

TABLA II

Respuesta al tratamiento aplicado

\begin{tabular}{cc} 
Tratamiento aplicado & $\begin{array}{c}\text { Casos que respondieron de forma } \\
\text { positiva al tratamiento (\%) }\end{array}$ \\
\hline Tintura de Propóleo & $80,0^{\mathrm{a}}$ \\
Clorhexidina & $3,4^{\mathrm{b}}$ \\
\hline
\end{tabular}

Letras distintas indican diferencias estadísticamente significativas $(P<0,05)$

indicando que el vehículo permitiría ejercer el efecto de la $C$ por mayor tiempo [16]. Asimismo, la capacidad adherente de TP es una de las características que permiten permanecer por mayor tiempo al producto en contacto con la pieza dental y ejercer su efecto bactericida. C en solución acuosa, parece perder su efecto conforme pasa el tiempo de preparación [10].

En la TABLA III, se describe el resultado del tratamiento haciendo referencia al tipo de microorganismo aislado. La eficacia del tratamiento cuando existe monoinfección, el efecto bactericida de TP es más efectivo respecto a $C(P<0,05)$. El resultado sobre la asociación entre Staphylococcus spp. y Corynebacterium spp. muestra una pérdida significativa en la eficacia de TP sobre estos microorganismos, pudiendo complicar en estos casos su resolución. $\mathrm{C}$ no tuvo ningún efecto independientemente del tipo de microorganismo o sus asociaciones, lo que indica la ineficacia de este tratamiento en pacientes caninos con EPC. 
TABLA III

Eficacia del tratamiento sobre los microorganismos y sus asociaciones, aisladas en los pacientes sujetos a estudio $(n=30)$

\begin{tabular}{ccc}
\hline Microorganismo & Propóleo & Clorhexidina \\
\hline Staphylococcus spp. & $94,11^{\mathrm{a}}$ & $0^{\mathrm{b}}$ \\
Corynebacterium spp. & $100,0^{\mathrm{a}}$ & $0^{\mathrm{b}}$ \\
Staphylococcus spp. | Corynebacterium spp. & $28,58^{\mathrm{a}}$ & $0^{\mathrm{b}}$ \\
Staphylococcus spp. | Lactobacillus spp. & $100,0^{\mathrm{a}}$ & $0^{\mathrm{b}}$ \\
Staphylococcus spp. | Enterobacterias & $100,0^{\mathrm{a}}$ & $0^{\mathrm{b}}$ \\
Staphylococcus spp. | Corynebacterium spp. | Enterobacterias & 0 & $0^{-}$ \\
\hline
\end{tabular}

Letras distintas en la misma fila indican diferencias estadísticamente significativas $(P<0,05)$

Hubo resultados similares cuando se analizó la eficacia del TP y $\mathrm{C}$, en los casos de EPC leve, moderada y grave. Estos resultados corroboran lo indicado anteriormente, donde TP ha sido utilizado en la aplicación terapéutica en diferentes patologías, tales como caries, aftas bucales y cicatrizaciones en el ser humano [17], siendo TP eficaz frente a agentes infecciosos, mostrando ser un buen antibacteriano natural, lo cual sugiere su uso en los casos de EPC $[2,11]$.

\section{CONCLUSIONES}

Los microorganismos que se hallaron con mayor frecuencia en la EPC por orden de importancia fueron: Staphylococcus spp. y Corynebacterium spp. Además, de forma aislada Lactobacillus spp. y Enterobacterias. De acuerdo con los resultados obtenidos en el presente estudio, TP ejerce un efecto superior a la solución acuosa de C. TP proporcionó los mejores resultados terapéuticos independientemente del grado de evolución de la enfermedad, del microorganismo y asociación bacteriana diagnosticada.

\section{REFERENCIAS BIBLIOGRÁFICAS}

[1] AHN, M.R.; KUMAZAWA, S.; HAMASAKA, T.; BANG, K.S.; NAKAYAMA, T. Antioxidant activity and constituents of propolis collected in various areas of Korea. Agric. Food Chem. 52 (24):7286-92. 2004.

[2] AN, H.J.; LEE, W.R.; KIM, K.H.; KIM, J.Y.; LEE, S.J.; HAN, S.M.; LEE, K.G.; LEE, C.K.; PARK, K.K. Inhibitory effects of bee venom on Propionibacterium acnes-induced inflammatory skin disease in an animal model. Int. J. Mol. Med. 34 (5):1341-8. 2014.

[3] ALBUQUERQUE, C.; MORINHA, F.; REQUICHA, J.; MARTINS, T.; DIAS, I.; GUEDES-PINTO, H.; BASTOS, E.; VIEGAS, C. Canine periodontitis: the dog as an important model for periodontal studies. Vet. J. 191 (3):299-305. 2012.

[4] DAVIS, I.J.; WALLIS, C.; DEUSCH, O.; COLYER, A.; MILELLA, L.; LOMAN, N.; HARRIS, S. A cross-sectional survey of bacterial species in plaque from client owned dogs with healthy gingiva, gingivitis or mild periodontitis. PLoS One. 8 (12): 1-12. 2013.
[5] DAHLÉN, G.; CHARALAMPAKIS, G.; ABRAHAMSSON, I.; BENGTSSON, L.; FALSEN, G. Predominant bacterial species in subgingival plaque in dogs. J. Periodontal Res. 47 (3): 354-64. 2012.

[6] GIL, M.; PERELLI, A.; ALVARADO, R.; ARIAS, Y.; BLUMENTHAL, E. Actividad bacteriostática y bactericida de la tintura de propóleos sobre bacterias enteropatógenas. Rev. Fac. Cs. Salud. 16 (3): 21-25. 2012.

[7] HOLMSTROM, S.E. Veterinary dentistry in senior canines and felines. Vet. Clin. North Am. Small Anim. Pract. 42 (4): 793-808. 2012.

[8] HOTWANI, K.; BALIGA, S.; SHARMA, K. Phytodentistry: use of medicinal plants. J. Complement Integr. Med. 11 (4): 233-51. 2014

[9] INSTITUTO NACIONAL DE METEOROLOGÍA E HIDROLOGÍA (INAMHI), Ecuador. 2014. Anuario Meteorológico, N 51-2011. En Línea: https://www.gob.ec/inamhi 27-05-2021.

[10] KIRAN, M.; ARPAK, N.; UNSAL, E.; ERDOGAN, M.F. The effect of improved periodontal healthon metabolic control in type 2 diabetes mellitus. J. Clin. Periodontol. 32: 266-272. 2005.

[11] KUMAZAWA, S.; BONVEHÍ, J.S.; TORRES, C.; MOK-RYEON, A.; BERMEJO, F.J. Chemical and functional characterisation of propolis collected from East Andalusia (southern Spain). Phytochem. Anal. 24 (6): 608-15. 2013.

[12] LAPENNA, M.; MEDINA, G.; DÍAZ, L.; AGUILLÓN, K.; MARÍN, H. actividad bactericida y fungicida de algunas plantas utilizadas en la medicina tradicional venezolana. Rev. Inst. Nac. Hig. Rafael Rangel. 34 (1): 6-9. 2003.

[13] LEVI, J.R.; BRODY, R.M.; MCKEE-COLE, K.; PRIBITKIN, E.; O'REILLY, R. Complementary and alternative medicine for pediatric otitis media. Int. J. Pediatr. Otorhinolaryngol. 77 (6): 926-31. 2013.

[14] LOW, S.B.; PEAK, R.M.; SMITHSON, C.W.; PERRONE, J.; GADDIS, B.; KONTOGIORGOS, E. Evaluation of a topical gel containing a novel combination of essential oils and antioxidants for reducing oral malodor in dogs. Am. J. Vet. Res. 75 (7): 653-7. 2014. 
[15] MARSHALL, M.D.; WALLIS, C.V.; MILELLA, L.; COLYER, A.; TWEEDIE, A.D.; HARRIS, S. A longitudinal assessment of periodontal disease in 52 Miniature Schnauzers. BMC Vet. Res. 10 (1):166. 2014.

[16] MUMMOLO, S.; TIERI, M.; TECCO, S.; MATTEI, A.; ABANI, F.; GIUCA, M.; MARZO, G. Clinical evatution of salivary indices and levels Streptococcus mutans and Lactobacillus in patients treated with Occlus Guide. Europ. J. Paedriat. Dent. 15: 367-370. 2014.

[17] PREMOLI, G.; LAGUADO, P.; DÍAZ, N.; ROMERO, C.; VILLARREAL, J.; GONZÁLEZ,A. Uso del propóleo en odontología. A. Odont. Vzlana. 48 (2): 1-13. 2010.
[18] RESTREPO, A.I; VELASCO, S.C.; FRANCO, L. Evolution of Explanatory Models of Periodontal Disease Pathogenesis. Rev. Estomat. 17 (2): 52-59. 2009.

[19] SCHMIDLIN, P.R.; ENGLISH, H.; DUNCAN, W.; BELIBASAKIS, G.N.; THUMHEER, T. Antibacterial potential of Manuka honey against three oral bacteria in vitro. Swiss Dent. J. 124 (9): 922-924. 2014

[20] STATISTICAL PACKAGE FOR THE SOCIAL SCIENCES (SPSS). SPSS Inc. Headquarters, 733 S. Wacker Drive, 11th floor. Chicago, USA. 2005. 
